\title{
Effects of daily wild blueberry (poly)phenol consumption on vascular function in a healthy elderly population: the BluFlow study
}

\author{
E. Wood ${ }^{1}$, S. Hein ${ }^{2}$, F. Fernandes ${ }^{3}$, C. Williams ${ }^{2}$ and A. Rodriguez-Mateos ${ }^{1}$ \\ ${ }^{1}$ Department of Nutritional Sciences, School of Life Course Sciences, King's College London, UK, \\ ${ }^{2}$ School of Psychology and Clinical Language Sciences, University of Reading, UK and \\ ${ }^{3}$ Department of Medical Engineering and Physics, King's College Hospital NHS Foundation Trust, UK
}

Increasing evidence from both epidemiological studies and randomised-controlled trials (RCT's) suggests that (poly)phenol-rich foods, such as blueberries, can improve vascular function, reducing the risk of developing cardiovascular diseases ${ }^{(1)}$. In addition, (poly)phenols are thought to improve beneficial gut bacteria and improve gut microbial diversity ${ }^{(2)}$. There is limited evidence in older populations, already at increased disease risk, and whether blueberry (poly)phenols can improve such health outcomes ${ }^{(3)}$. In this study, we aim to investigate whether daily wild blueberry supplementation can lead to changes in vascular function (measured as flow-mediated dilation, FMD), cerebral blood flow (CBF) and improve the gut microbiome in a cohort of healthy elderly individuals.

A parallel, double blind, placebo controlled RCT was conducted in 61 healthy individuals, aged 65-80. The impact of wild blueberry (poly)phenols on FMD, active and resting CBF using transcranial doppler ultrasound, and $24 \mathrm{~h}$ blood pressure (BP) was assessed at baseline and 12-weeks following daily consumption of $26 \mathrm{~g}$ freeze-dried blueberry powder, containing $264 \mathrm{mg}$ anthocyanins (equivalent to $178 \mathrm{~g}$ fresh wild blueberries), or a macronutrient and micronutrient matched control drink (0 $\mathrm{mg}$ anthocyanins). Faecal samples were collected to measure changes in the gut microbiome. Blood and $24 \mathrm{~h}$ urine samples were also collected to analyse (poly)phenol metabolites. Linear mixed model analysis was performed as the statistical method.

FMD improved by $0.86 \%(\mathrm{f}=32.8, \mathrm{p}<0.001)$ in the blueberry group compared with the placebo group after 12-weeks daily consumption. Systolic $24 \mathrm{~h}$ ambulatory BP decreased by $-3.59 \mathrm{mmHg}(\mathrm{f}=4.6, \mathrm{p}=0.037)$ in the blueberry group compared to the placebo group. No other vascular outcome changed significantly following daily blueberry consumption. Results for the gut microbiome changes and with the plasma and urine (poly)phenol concentrations will be available at the time of this conference.

Daily consumption of wild blueberry (poly)phenols improves FMD and blood pressure in a healthy elderly population. This suggests that daily blueberry consumption can improve vascular function in an at-risk population. However, other vascular outcomes such as pulse wave velocity and cerebral blood flow did not change. More research is needed to investigate whether blueberries can improve such outcomes in elderly individuals with pre-existing cardiovascular risk factors such as hypertension.

\section{References}

1. X. Wang, Y. Y. Ouyan, J. Liu and G. Zhao (2014) Br J Nutr, 111, 1-11.

2. Bolca S, Van de Wiele T, Possemiers S (2013) Curr Opin Biotech, 24, 250-225.

3. Wood E, Hein S, Heiss C, Williams C, Rodriguez-Mateos A (2019) Food \& Function, 10, 7621-7633. 